\title{
Fluid type influences acute hydration and muscle performance recovery in human subjects
}

\author{
Preston R. Harris ${ }^{1}$, Douglas A. Keen², Eleni Constantopoulos ${ }^{2,3}$, Savanna N. Weninger ${ }^{2}$, Eric Hines ${ }^{2,3}$,
} Matthew P. Koppinger ${ }^{1}$, Zain I. Khalpey ${ }^{4}$ and John P. Konhilas ${ }^{2,3^{*}}$

\begin{abstract}
Background: Exercise and heat trigger dehydration and an increase in extracellular fluid osmolality, leading to deficits in exercise performance and thermoregulation. Evidence from previous studies supports the potential for deep-ocean mineral water to improve recovery of exercise performance post-exercise. We therefore wished to determine whether acute rehydration and muscle strength recovery was enhanced by deep-ocean mineral water following a dehydrating exercise, compared to a sports drink or mountain spring water. We hypothesized that muscle strength would decrease as a result of dehydrating exercise, and that recovery of muscle strength and hydration would depend on the type of rehydrating fluid.
\end{abstract}

Methods: Using a counterbalanced, crossover study design, female $(n=8)$ and male $(n=9)$ participants performed a dehydrating exercise protocol under heat stress until achieving 3\% body mass loss. Participants rehydrated with either deep-ocean mineral water (Deep), mountain spring water (Spring), or a carbohydrate-based sports drink (Sports) at a volume equal to the volume of fluid loss. We measured relative hydration using salivary osmolality $\left(\mathrm{S}_{\mathrm{osm}}\right)$ and muscle strength using peak torque from a leg extension maneuver.

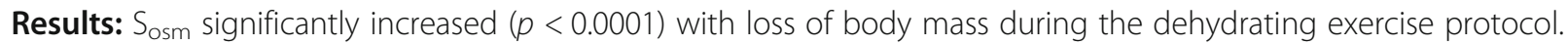
Males took less time $(90.0 \pm 18.3 \mathrm{~min} ; P<0.0034)$ to reach $3 \%$ body mass loss when compared to females $(127.1$ $\pm 20.0 \mathrm{~min}$ ). We used a mono-exponential model to fit the return of $S_{\text {osm }}$ to baseline values during the rehydrating phase. Whether fitting stimulated or unstimulated $S_{\text {osm, }}$ male and female participants receiving Deep as the hydrating fluid exhibited the most rapid return to baseline $S_{\text {osm }}(p<0.0001)$ regardless of the fit parameter. Males compared to females generated more peak torque $(p=0.0005)$ at baseline $(308.3 \pm 56.7 \mathrm{Nm}$ vs $172.8 \pm 40.8 \mathrm{Nm}$, respectively) and immediately following $3 \%$ body mass loss $(276.3 \pm 39.5 \mathrm{Nm}$ vs $153.5 \pm 35.9 \mathrm{Nm}$ ). Participants experienced a loss. We also identified a significant effect of rehydrating fluid and sex on post-rehydration peak torque $(p<0.0117)$.

Conclusion: We conclude that deep-ocean mineral water positively affected hydration recovery after dehydrating exercise, and that it may also be beneficial for muscle strength recovery, although this, as well as the influence of sex, needs to be further examined by future research.

Trial registration: clincialtrials.gov PRS, NCT02486224. Registered 08 June 2015.

Keywords: Dehydration, Exercise, Deep sea water, Humans, Sweat

\footnotetext{
* Correspondence: konhilas@arizona.edu

2Department of Physiology, University of Arizona, Tucson, AZ 85721, USA

${ }^{3}$ Sarver Molecular Cardiovascular Research Program, University of Arizona,

Tucson, AZ 85724, USA

Full list of author information is available at the end of the article
}

(c) The Author(s). 2019 Open Access This article is distributed under the terms of the Creative Commons Attribution 4.0 International License (http://creativecommons.org/licenses/by/4.0/), which permits unrestricted use, distribution, and reproduction in any medium, provided you give appropriate credit to the original author(s) and the source, provide a link to the Creative Commons license, and indicate if changes were made. The Creative Commons Public Domain Dedication waiver (http://creativecommons.org/publicdomain/zero/1.0/) applies to the data made available in this article, unless otherwise stated. 


\section{Background}

The volume of total body water in humans represents approximately $60-70 \%$ of total body weight in men and $50-60 \%$ of body weight in women, with some variability primarily due to differences in body composition [1]. Many factors impact total fluid volume including diet (water and sodium intake), environmental temperature, evaporation, activity level, and certain disease states. Humans have a remarkable capacity to maintain constant osmolality of extracellular fluid (ECF) through both behavioral responses and physiological mechanisms that restore ECF to homeostatic values [2]. Still, loss of body water, or dehydration, impairs normal physiologic function. Dehydration increases cardiovascular strain by reducing blood volume through fluid loss, thereby decreasing stroke volume, and increasing heart rate. When core temperature increases as a result of exercise and dehydration, elevated skin blood flow displaces blood away from the central blood volume, exacerbating cardiovascular strain [3]. In addition to this increase in cutaneous blood flow, sweat production increases in an effort to dissipate heat to the environment through evaporative cooling [4].

Sweat glands produce a hypotonic fluid in relation to plasma by drawing fluid from the ECF concomitant with reabsorption of sodium and chlorine via the cystic fibrosis transmembrane protein (CFTR) channels [5]. This results in both a decrease in total body water volume and an increase in ECF osmolality [6]. Governed by hydrostatic, osmotic and oncotic pressures, the increase in ECF osmolality triggers water movement from plasma and intracellular stores to restore osmolality in the interstitial fluid compartment $[1,2]$. Osmotic fluctuations, if severe enough, can have serious health consequences, such as weakness, cardiac arrest, spasticity, coma, seizures, and death [2, 7]. Furthermore, dehydration impairs thermoregulation [8] and exercise performance, independently of thermal, dietary, or metabolic stressors $[3,9]$. Exercising in a hot environment amplifies dehydration, exacerbates the above effects, and accelerates performance deficits $[3,10,11]$.

An obvious key to preventing the detrimental impact of fluid loss on exercise performance is to replenish fluid deficit through oral consumption [5]. Although the American College of Sports Medicine Guidelines on Nutrition and Athletic Performance recommend the amount of fluid intake, there is no clear endorsement regarding the specific type of rehydrating fluid [12]. A recent study shows that deep-ocean water taken from the coast of Hualien, Taiwan at a depth of $662 \mathrm{~m}$ improves recovery following a dehydrating exercise, evidenced by accelerated recovery of aerobic capacity, increased lower-body muscle power performance, and significantly reduced levels of exercise-induced muscle damage markers compared to participants drinking purified tap-water [13]. In another study, deep-ocean mineral water was shown to increase the exercise performance of gerbils, compared to distilled water, measured by retention rates during a 90-min treadmill exercise [14]. Considering the established connection between hydration status and exercise performance, these data suggest that deep-ocean mineral water may provide optimal rehydration for performance recovery following high-intensity exercise. Accordingly, data from our previous study [15] suggested that Kona Deep-ocean mineral water had the potential to improve lower-body muscle strength as well as acute rehydration rate after dehydrating exercise.

Deep ocean mineral water has a mineral composition that differs significantly from that of water found on or near the surface, often containing much higher amounts of sodium, potassium, chloride, magnesium, and various other trace minerals which are not found in surface water sources. Although mineral profiles of deep ocean mineral water differ by specific site, it is this unique mineral composition that we believe is responsible for the effects described above, though we do not propose a mechanism here.

The objective of this study was to improve and expand upon the observations from our previous work, regarding the impact of fluid type on rehydration and muscle performance recovery following rehydration at the completion of a dehydrating exercise protocol under heat stress. We hypothesized that rehydration with Kona Deep ${ }^{\circ}$ ocean mineral water will accelerate the rate of acute rehydration, and will improve muscle strength recovery compared to Gatorade ${ }^{\circledR}$ or mountain spring water. Secondarily, we make observations on potential sex differences in these parameters.

\section{Methods \\ Participants}

Participants eligible for this study included females and males ages 20-25 years, who were nonsmokers and were well-conditioned with moderate to excellent aerobic capacity. Participants were free from medication, stimulants, dietary supplements (including vitamins/minerals, amino acids, and herbal supplements, as defined by the FDA), or major health-related issues, as determined by a University of Arizona Health History Screening Questionnaire (UAHHSQ). Age-matched female and male study participants meeting eligibility criteria were further selected for equivalent fitness levels based on a self-reported $3-6 \mathrm{~h}$ of athletic conditioning per week. The study population engaged in primarily dynamic activities including cycling, running, hockey, soccer, and triathlons. With a conservative estimate of salivary osmolality at baseline $(100 \mathrm{mmol} / \mathrm{Kg})$ compared to peak 
(150 $\mathrm{mmol} / \mathrm{Kg}$ ) during the experimental protocol with a standard deviation of 30 , sample size was determined using a power of 0.8 at $\alpha=0.05$ (6 or greater per group). This was also confirmed using power analysis of mean population serum and urinary osmolality. All participants provided consent under protocols adhering to guidelines approved by the Institutional Review Board at the University of Arizona and in accordance with the Declaration of Helsinki. Participants were asked to follow a normal diet while avoiding foods high in sodium $24 \mathrm{~h}$ prior to study initiation, and to avoid any exercise $24 \mathrm{~h}$ prior to each trial. Dietary data was not collected. In addition, participants were required to abstain from alcohol consumption $36 \mathrm{~h}$ prior to each exercise trial, as alcohol has diuretic effects, and may confound observed effects of dehydration. Female participants were asked to complete the study early in their menstrual cycle to avoid the potentially confounding issue of fluid retention. Participants were also instructed to begin each trial in a similarly hydrated state (confirmed by consistent baseline salivary osmolality at the start of each trial).

\section{Study design}

We used a counterbalanced, crossover study design in which participants $(n=17)$ were randomized to begin in one of 3 experimental groups: Kona Deep ${ }^{\circ}$ deep-ocean mineral water (Deep), Gatorade ${ }^{\bullet}$ sports drink (Sports), or Arrowhead mountain spring water (Spring) (See Table 1 for fluid nutrient comparison). Each participant was required to complete the dehydrating exercise protocol 3 times, one trial for each hydration fluid, each separated by a period of at least $48 \mathrm{~h}$, to give participants adequate recovery time. After the first trial, participants were randomized to complete the second arm of the study, hydrating with one of the two remaining hydrating fluids. During the third trial, participants rehydrated with the last remaining fluid.

\section{Dehydration and hydration protocols}

A graphical summary of the Dehydration and Hydration Protocol is illustrated in Fig. 1. Participants were asked to remove any excess or loose clothing including shirts, athletic pants, shoes, and socks. Participants were instrumented with a Polar $^{\text {rix }}$ heart rate monitor allowing continuous monitoring of heart rate. Baseline measurements of heart rate, body weight (using a digital scale) and tympanic temperature (Braun ThermoScan ${ }^{\circ} \mathrm{PRO}$ 4000) were collected prior to the initiation of the exercise dehydration protocol. In addition, stimulated and unstimulated saliva samples (detailed below) were taken to establish baseline osmolality values and to ensure all participants were similarly hydrated at the start of each trial. Next, participants initiated exercise using a Monark $^{\circ}$ stationary cycle under moderate heat stress using heat lamps to provide warm conditions of about $30^{\circ} \mathrm{C}$, since sweat rate is greatest at warm ambient temperatures [16]. Participants were instructed to sustain about $60 \%$ of maximum heart rate and maintain $150-200$ watts on the stationary bicycle at a cadence greater than 70 rpm. Participants self-monitored and adjusted watts by varying cycle speed or resistance. Exercise was continued for $15 \mathrm{~min}$, at which time participants were asked to remove any excess or loose clothing as above, and towel-dry for data collection (body weight, heart rate, temperature, saliva samples). Participants were not allowed to evacuate or intake any fluids during the exercise protocol. This protocol was repeated at 15-min intervals until target dehydration, indicated by a body mass loss of $3 \%$ was achieved. Studies have indicated that greater than $2 \%$ body mass loss by dehydration results in a significant exercise deficit $[3,10,13,14]$.

When $3 \%$ body mass loss was reached, participants rehydrated by consuming a volume of fluid (Deep, Sports, or Spring) equal to their body mass loss, assuming that each $1 \mathrm{~L}=1 \mathrm{~kg}$. Rehydration occurred in two phases. In the first phase, participants consumed

Table 1 Fluid comparison of selected nutrients

\begin{tabular}{llll}
\hline Nutrient & Kona Deep ${ }^{\oplus}$ (deep-ocean mineral water) ${ }^{a}$ & Arrowhead (mountain spring water) $^{\mathrm{b}}$ & Gatorade $^{\oplus}$ (carbohydrate-based sports drink) $^{\mathrm{c}}$ \\
\hline Sodium & $85 \mathrm{mg} / \mathrm{L}$ & $3 \mathrm{mg} / \mathrm{L}$ & $450.9 \mathrm{mg} / \mathrm{L}$ \\
Chloride & $150 \mathrm{mg} / \mathrm{L}$ & $0-12 \mathrm{mg} / \mathrm{L}$ & $408.3 \mathrm{mg} / \mathrm{L}$ \\
Potassium & $4 \mathrm{mg} / \mathrm{L}$ & $0-2.9 \mathrm{mg} / \mathrm{L}$ & $126.8 \mathrm{mg} / \mathrm{L}$ \\
Magnesium & $4.3 \mathrm{mg} / \mathrm{L}$ & $1.4 \mathrm{mg} / \mathrm{L}$ & $0 \mathrm{mg} / \mathrm{L}$ \\
Calcium & $1.4 \mathrm{mg} / \mathrm{L}$ & $4 \mathrm{mg} / \mathrm{L}$ & $0 \mathrm{mg} / \mathrm{L}$ \\
Boron & $0.65 \mathrm{mg} / \mathrm{L}$ & $0 \mathrm{mg} / \mathrm{L}$ & $0 \mathrm{mg} / \mathrm{L}$ \\
Bromide & $540 \mu \mathrm{g} / \mathrm{L}$ & $3.1 \mu \mathrm{gg} / \mathrm{L}$ & $0 \mu \mathrm{g} / \mathrm{L}$ \\
Chromium & $2.2 \mu \mathrm{g} / \mathrm{L}$ & $0 \mu \mathrm{g} / \mathrm{L}$ & $0 \mu \mathrm{g} / \mathrm{L}$ \\
Carbohydrates & $0 \mathrm{~g} / \mathrm{L}$ & $0 \mathrm{~g} / \mathrm{L}$ & $59.2 \mathrm{~g} / \mathrm{L}$ \\
\hline
\end{tabular}

${ }^{a}$ Values provided by Kona Deep ${ }^{\oplus}$ Corporation via a 3rd party laboratory report. ${ }^{b}$ Values are minimum ranges based on Arrowhead 2016 Water Analysis Report at www.nestle-watersna.com. 'Values calculated based on amounts reported for Gatorade ${ }^{\circledast}$ Thirst Quencher at www.Gatorade.com. Values were not validated in house 


\section{Dehydration Protocol:}

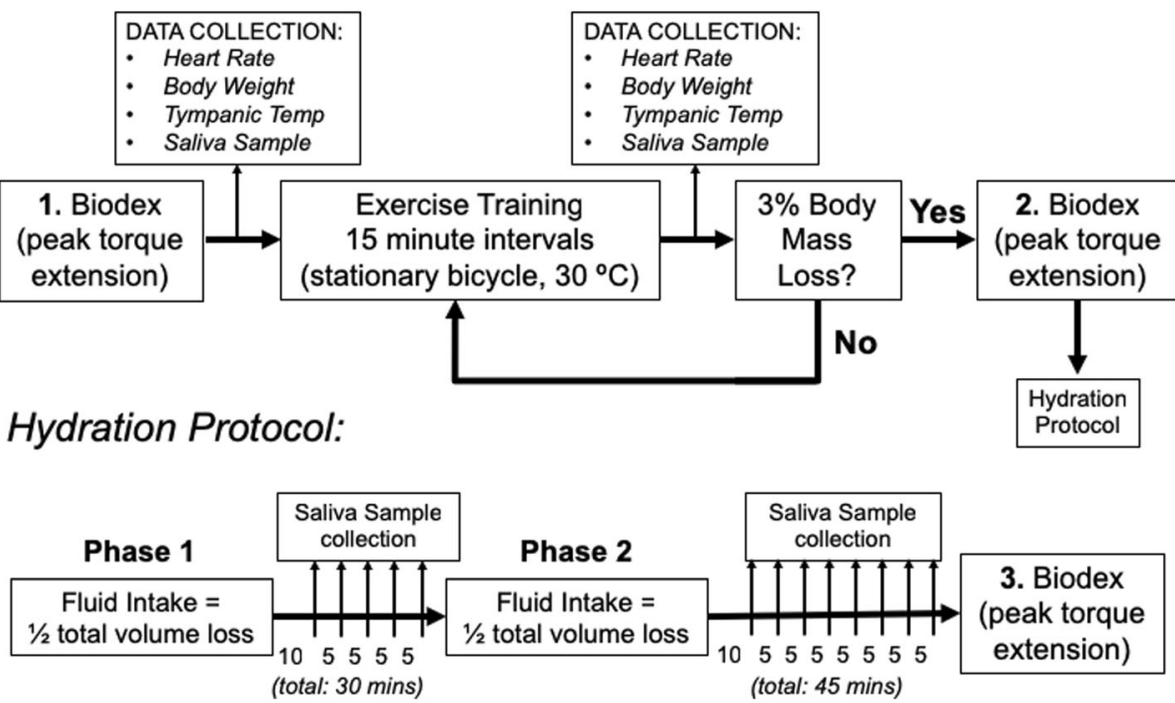

Fig. 1 Experimental design and protocol. Dehydration Protocol: Euhydrated participants were randomly assigned in a counterbalanced fashion to one of three groups (Deep, Sports, or Spring). Prior to data collection, participants executed 1 of 3 peak torque extension maneuvers to obtain a baseline value. Following peak torque extension, parameters were collected (indicated in the panel labeled DATA COLLECTION) and exercise was initiated using a stationary bicycle under moderate heat stress $\left(32-35^{\circ} \mathrm{C}\right)$. Exercise was continued for 15 min followed by collection of parameters. If participants did not achieve $3 \%$ body mass loss, exercise was reinitiated for another $15 \mathrm{~min}$. This cycle was continued until participants lost a minimum 3\% of body mass and participants were not allowed to evacuate or intake any fluids. Upon completion of the Dehydration Protocol, participants immediately executed the second (2) of 3 peak torque extension maneuvers to obtain a post-exercise value and transitioned to the Hydration protocol. Hydration protocol: Participants rehydrated with 1 of 3 fluids, in 2 phases. Phase 1: Participants consumed fluids at $1 / 2$ of the total volume lost. 10 min following fluid intake a saliva sample was collected. Sample collection continued at 5-min intervals until 30 min from the time of fluid intake. Phase 2: The remaining amount ( $1 / 2$ of the total volume lost) of fluid was ingested followed by saliva sample collection 10 min later. Saliva collection continued at 5-min intervals until $45 \mathrm{~min}$ from the second fluid intake. Immediately following the final saliva collection, participants executed the third [3] of 3 peak torque extension maneuvers to obtain a post-hydration value.

one-half of the total volume lost. Stimulated and unstimulated saliva samples were collected starting at 10 min after this initial rehydration phase (to avoid the cofounding effect on salivary osmolality due to oral rinse) [17], and at each subsequent 5-min interval, for $30 \mathrm{~min}$. After this 30 -min time period, the second phase occurred, in which participants consumed the remainder of the fluid. Saliva samples were taken starting at $10 \mathrm{~min}$ after this second rehydration phase, and at each subsequent 5-min interval, for $45 \mathrm{~min}$.

\section{Lower body muscle performance}

Peak torque of the left knee extensors, measured on a Biodex $^{\text {ra }}$ System 3 dynamometer, was used as a measure of lower body muscle performance. As illustrated in Fig. 1 , a peak torque extension maneuver was executed three times during the Dehydration and Hydration Protocols: 1) baseline - prior to exercise, 2) post-exercise - following loss of $3 \%$ body mass, and 3) post-hydration after the final saliva collection ( $75 \mathrm{~min}$ post-exercise). Participants performed a series of 3 maximal contractions of the left knee extensors at a rotational velocity of 60 degrees per second using the Biodex ${ }^{\mathrm{Tw}}$. To maintain consistency, participants performed this test oriented in the same position, and using the same hand grips for support during each of the measurements. Participants were also vigorously encouraged to exert maximal effort on each measurement by the same individuals. To familiarize participants with the Biodex ${ }^{\mathrm{Tm}}$ machine, they were allowed a "practice" period prior to each measurement to move their leg at will, and become accustomed to the speed of movement. When the participants were comfortable and ready to perform the measured test, they indicated this to the machine by holding their leg in a fully contracted position for several seconds, signaling the measurement process detailed above to proceed.

\section{Salivary osmolality}

Hydration status was monitored using salivary osmolality. Several different measurements can be used to assess hydration, including serum, saliva, and urine osmolality, and urine volume and specific gravity, and the most appropriate measurement depends on the mode of dehydration, and the frequency of the measurement. Previous studies have demonstrated that, for repeated measurements during active dehydration (i.e. exercise) in the 
heat, salivary osmolality is an accurate, non-invasive method to measure ECF osmolality [18].

Saliva was collected from the oral cavity, first as a passive expectorant (unstimulated) [17, 19], and then following mechanical (stimulated) orofacial movement (chewing on a cotton swab). Stimulated saliva samples were spun down in a centrifuge for 10 min to collect saliva from the cotton swabs. All samples, both stimulated and unstimulated were then vortexed to homogenize the samples. Salivary osmolality was measured using a dedicated vapor pressure osmometer (Wescor VAPRO ${ }^{\circ}$ 5600 ) by the same three individuals, using $10 \mu \mathrm{l}$ of each sample, and each sample was run in triplicate. This was done immediately after sample collection to prevent sample spoilage. In addition to daily calibrations, the osmometer was calibrated prior to each new biological sample.

\section{Data and statistical analysis}

All values are presented as mean (SD). Body Mass Index (BMI) was calculated using the following equation:

$$
\mathrm{BMI}=\text { body weight }(\text { in } \mathrm{kg}) / \text { height }(\text { in meters })^{2}
$$

Body Surface Area (BSA) was calculated based on the following equation [20]:

$$
\text { BSA }\left(\mathrm{m}^{2}\right)=\sqrt{ }(\text { Height }(\text { in } \mathrm{cm}) \mathrm{x} \text { body weight }(\text { in } \mathrm{kg})) / 3600
$$

To compare heart rate, body weight (BW), BMI, BSA, and tympanic temperature at baseline and peak, the measured values in each individual were averaged across the three arms of the study. The same method was used to calculate mean values for time to $3 \%$ body mass loss, sweat rate, baseline and post-exercise peak torque. Salivary osmolality $\left(\mathrm{S}_{\mathrm{osm}}\right)$ was plotted against percent body mass loss; body mass loss was calculated as the difference in body mass after completion of the dehydrating exercise, from body mass at trial initiation. This value was divided by body mass at trial initiation and expressed as a percentage. For each individual, $\mathrm{S}_{\text {osm }}$ against body mass loss (\%) was fit by linear regression. Differences in the slopes of the regression lines between the groups were calculated using one-way analysis of variance (ANOVA) with Bonferroni post hoc correction for multiple comparisons. The return of $S_{\text {osm }}$ to baseline during the Hydration Protocol was best fit by a mono-exponential (one-phase decay) model where,

$$
\begin{array}{r}
\text { Sosm }=(\text { Sosm }(\text { peak })-\text { Sosm }(\text { baseline }))^{\mathrm{Kt}} \\
+ \text { Sosm (baseline })
\end{array}
$$

$\mathrm{S}_{\text {osm }}=$ Salivary osmolality; $\mathrm{S}_{\text {osm(baseline) }}=$ Salivary osmolality at baseline (approximate; calculated as plateau phase of best fit model); $S_{\text {osm(peak) }}=$ Salivary osmolality at peak ( $\mathrm{S}_{\mathrm{osm}}$ at end of Dehydration Protocol).
Time was adjusted such that the time at $S_{\text {osm(peak) was }}$ set to $t=0$ in order to normalize fit parameters. $K=$ rate constant, tau $(\tau)=1 / K$, and $\tau_{1 / 2}=$ half-time $S_{\text {osm }}$ recovery. Performance loss was calculated as percent loss from baseline; performance recovery was calculated as a percentage of baseline peak torque where post-exercise peak torque was divided by baseline peak torque and expressed as a percentage (Peak Torque post-ex $/$ Peak Torque $_{\text {base }} \mathrm{X}$ 100).

We used $p$ values of $<0.05$ to indicate statistical significance. Statistical calculations were calculated using commercially available software (GraphPad Prism version 5.0 for Mac OS X). Differences between averaged age, height, heart rate, BW, BMI, BSA, tympanic temperature, time to $3 \%$ body mass loss and sweat rate were determined using 2-way ANOVA with post-hoc Bonferroni analysis. All other comparisons were completed using a repeated measures 2-way ANOVA followed by a post-hoc Bonferroni analysis. No non-parametric tests were necessary, as all data were normally distributed.

\section{Results}

\section{Age and body Morphometrics}

At the time of the study, female participants $(n=8)$ were $22.1 \pm 2.1$ years of age, which was not different from male participants $(n=9)$ who were $23.6 \pm 2.2$ years of age. Female participants were significantly less in height when compared to male counterparts $(167.6 \pm 2.9 \mathrm{~cm}$ vs $181.1 \pm 4.6 \mathrm{~cm})$. Considering the significant difference in height, BW $(65.2 \pm 10.2 \mathrm{~kg}$ vs. $76.0 \pm 8.6 \mathrm{~kg})$ and BSA $\left(1.74 \pm 0.11 \mathrm{~m}^{2}\right.$ vs $\left.1.95 \pm 0.14 \mathrm{~m}^{2}\right)$ were significantly less in females compared to males. However, this difference was eliminated in the calculated BMI; females had a BMI of $23.3 \pm 3.0 \mathrm{~kg} / \mathrm{m}^{2}$ while males had a BMI of 23.2 $\pm 3.4 \mathrm{~kg} / \mathrm{m}^{2}$.

\section{Heart rate and body temperature}

Although baseline heart rate trended higher in females $(85.8 \pm 6.4 \mathrm{bpm})$ compared to males $(80.4 \pm 16.3 \mathrm{bpm})$, the trend did not reach significance. For each 15 -min bout of exercise, we recorded peak heart rate and subsequently averaged these values to arrive at a single peak heart rate. We saw no significant impact of sex on peak heart rate, and no interaction between sex and exercise on peak heart rate.

Tympanic temperature as an indicator of core temperature was also recorded throughout the exercise protocol. Despite being subjected to exercise and moderate heat stress, both female $\left(97.9 \pm 0.5^{\circ} \mathrm{F}\right.$ vs. $\left.99.2 \pm 0.7^{\circ} \mathrm{F}\right)$ and male $\left(97.7 \pm 0.8^{\circ} \mathrm{F}\right.$ vs. $\left.99.3 \pm 0.7^{\circ} \mathrm{F}\right)$ participants maintained body temperature to a similar extent, displaying no significant increase in core temperature during exercise. 
Exercise and salivary osmolality

For each saliva sample (unstimulated and stimulated), we determined salivary osmolality $\left(\mathrm{S}_{\mathrm{osm}}\right)$ and plotted $\mathrm{S}_{\text {osm }}$ against the percent of body mass lost. For display purposes, we represent the data as binned samples \pm standard deviation (S.D.). Stimulated and unstimulated $\mathrm{S}_{\text {osm }}$ were significantly (positively) correlated with percent of body mass loss for both females and males. As illustrated in Fig. 2, $\mathrm{S}_{\text {osm }}$ progressively increased during exercise bouts paralleling lost body mass. The relationship of $\mathrm{S}_{\text {osm }}$ and percent body mass loss was not different between females and males.

No significant differences in baseline $S_{\text {osm }}$ among study groups based on fluid designation were detected, validating that participants began each arm of the three trials at a similar hydration level. Baseline $S_{\text {osm }}$ was not effected by sex in the stimulated (females $94.39 \pm 14.90$ vs males $113.00 \pm 63.84$ ), or the unstimulated (females $94.06 \pm 26.62$ vs males $95.51 \pm 33.27)$ saliva samples, and there was no interaction between study group designation and sex. Peak $\mathrm{S}_{\text {osm }}$ (stimulated and unstimulated) was taken as the value for $S_{\text {osm }}$ once participants reached at least 3\% body mass loss. Similar to baseline, peak $S_{\text {osm }}$ was not significantly impacted by either study group designation or sex in the stimulated (females $180.29 \pm 60.37$ vs males $256.96 \pm 104.57$ ), or the unstimulated (females $235.04 \pm 105.99$ vs males $297.14 \pm$ 102.39) saliva samples. Moreover, there was no significant interaction between these factors on peak $\mathrm{S}_{\text {osm }}$. At the completion of $3 \%$ body mass loss, all participants achieved a significant $(p<0.0001)$ increase in averaged $\mathrm{S}_{\text {osm }}$ over baseline values. However, this elevation in $\mathrm{S}_{\text {osm }}$ was not affected by the sex of the participant (Fig. 2d, e).

\section{Time to body mass loss}

We compared the expended time for participants to achieve a $3 \%$ body mass loss in each study arm (study group designation). Participants (female and male) did not differ in the time to achieve a $3 \%$ body mass loss between the 3 trials (Fig. 3a). We found a significant effect $(P<0.0125)$ of sex on time to achieve $3 \%$ body mass loss, with no interaction between study group designation and sex. Subsequent comparison of the mean values for each participant demonstrated that males took less time $(90.0 \pm 18.3 \mathrm{~min} ; P<0.0034)$ to reach the required body mass loss when compared to females $(127.1 \pm 20.0 \mathrm{~min})$ (Fig. 3b). Assuming that body mass loss during the exercise period was due to water loss, we calculated sweat rate using the absolute mass of lost body mass and the total time to reach 3\% body mass loss (Fig. 3c). Based on the averaged values of sweat rate for each participant, females $(15.3 \pm 3.2 \mathrm{ml} / \mathrm{min} ; p<0.0083)$ demonstrated lower sweat rates compared to males $(25.2 \pm 7.8 \mathrm{ml} / \mathrm{min})$, consistent with the greater time to achieve $3 \%$ body mass loss in the female group.

\section{Impact of fluid on $\mathrm{S}_{\text {osm }}$ recovery (rehydration)}

Peak $\mathrm{S}_{\text {osm }}$ steadily declined and returned to baseline $\mathrm{S}_{\mathrm{osm}}$ values before completion of the saliva collection
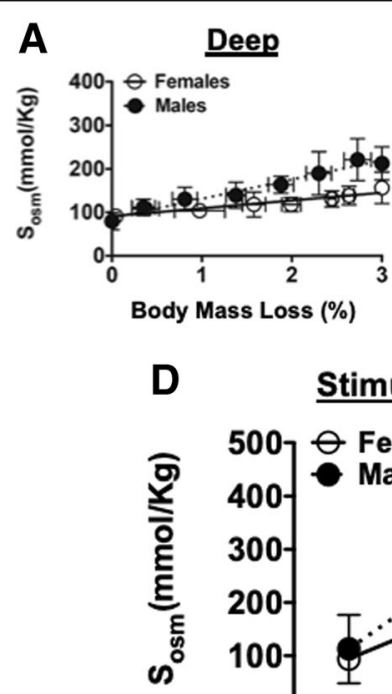

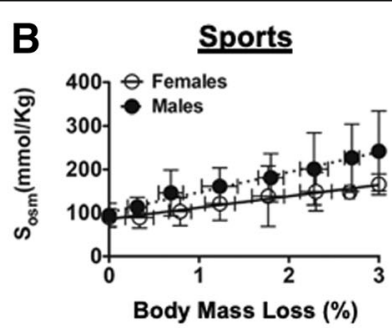

E

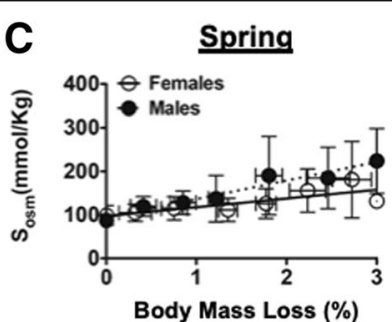

Unstimulated
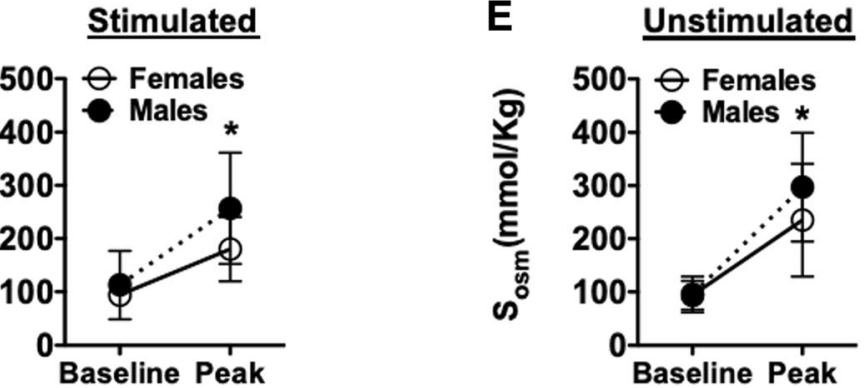

Fig. 2 Salivary osmolality as a function of body mass loss. Salivary osmolality $\left(\mathrm{S}_{\mathrm{osm}} \mathrm{mmol} / \mathrm{Kg}\right)$ was plotted as a function of change in body mass percentage in each of the three groups: Deep (a), Sports (b) or Spring (c) groups. Individual measures of salivary osmolality were averaged from the three trials. Salivary osmolality $\left(\mathrm{S}_{\mathrm{osm}} \mathrm{mmol} / \mathrm{Kg}\right)$ was significantly elevated $\left({ }^{*} p<0.0001\right.$; Females and Males) at Peak compared to Baseline collected as either Stimulated (d) or Unstimulated (e). No differences between Females and Males were detected, nor was there an interaction between sex and time point of data collection. Two-way ANOVA with post-hoc Bonferroni analysis. Data presented as mean \pm S.D 

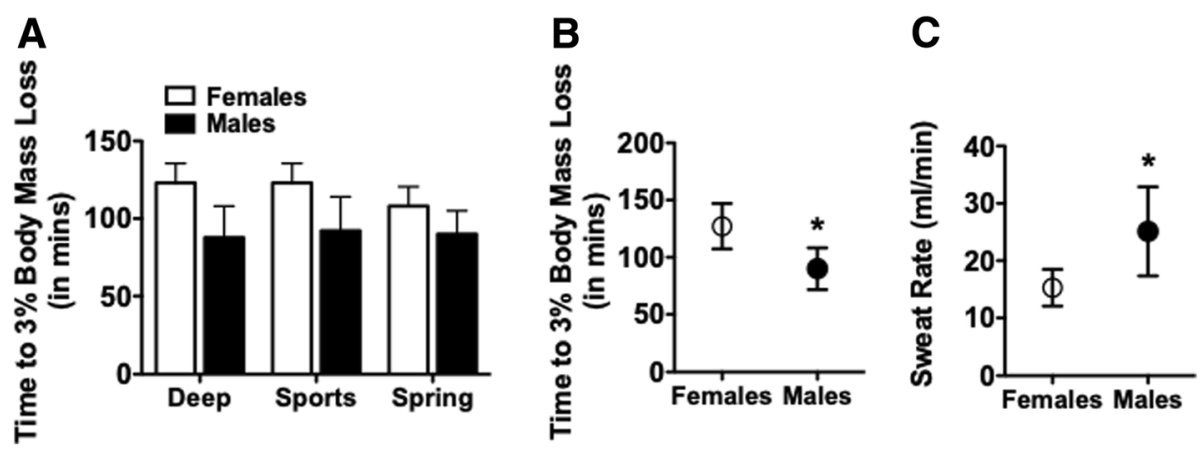

Fig. 3 Time required to achieve target dehydration indicated by a 3\% body mass loss. a Bar graph representation of the time required to reach a loss of body mass that was at least 3\% of starting body mass in the Deep, Sports and Spring groups for Females and Males. A repeated measures two-way ANOVA indicated no significant differences in Time to 3\% Body Mass Loss among the experimental groups but did find a significant effect of sex $(p<0.0125)$. b Averaged values across experimental groups for Time to 3\% Body Mass Loss in Females and Males. $\left(^{*} p<0.034 ;\right.$ Females vs. Males). c Averaged values across experimental groups for Sweat Rate ( $\mathrm{ml} / \mathrm{min}$; total volume lost in $\mathrm{ml}$ over total time to $3 \%$ body mass loss; ) in Females and Males. ( ${ }^{*} p<0.0083$; Females vs. Males). Two-way ANOVA with post-hoc Bonferroni analysis. Data presented as mean \pm S.D

time during the rehydration phase. We determined the rate constant $(\mathrm{K})$, time constant $(\tau)$, and the time constant half-life $\left(\tau_{1 / 2}\right)$ of this decay for each participant in each trial (Fig. 4a). We found a significant effect of rehydrating fluid $(p<0.0001)$ that was not impacted by sex. Post-hoc analysis revealed a significantly higher $\mathrm{K}$, and a significantly lower $\tau$, and $\tau_{1 / 2}$ in the Deep than in both the Sports and Spring groups in both females and males (Figs. 4b-d, values in Table 2). The same trend of significance was seen whether $S_{\text {osm }}$ was taken from the stimulated or the unstimulated samples.
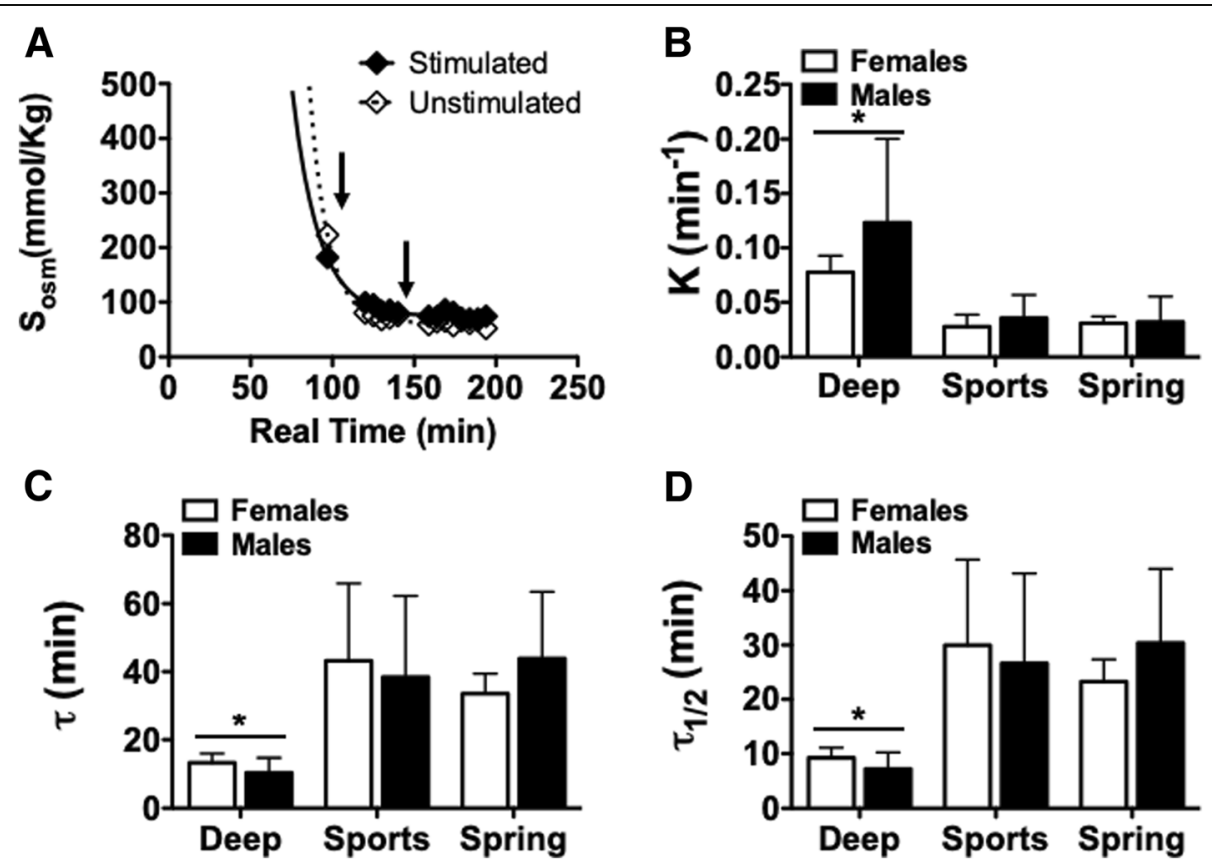

Fig. 4 Rate of salivary osmolality recovery during fluid hydration following dehydrating exercise protocol. Salivary osmolality was fit with a single exponential decay (one-phase decay) starting with peak salivary osmolality against real time. a representative one-phase decay fit to salivary osmolality recovery during fluid hydration. Fluid was ingested in two phases indicated by the arrows. $\mathbf{b}$-d Bar graph representation of one-phase decay fit parameters $\left(K, \operatorname{tau}(\tau)\right.$, and $\left.\tau_{1 / 2}\right)$ in Females and Males from the Deep, Sports and Spring groups. A repeated-measures two-way ANOVA determined a significant impact of fluid on rate parameters of hydration that was not impacted by sex. Post-hoc Bonferroni analysis indicated a significant difference in the Deep group compared to both Sports and Spring groups ( ${ }^{*} p<0.0001$ compared to Sports and Spring groups). Onephase decay equation: $S_{\text {osm }}=\left(S_{\text {osm(peak) }} S^{-} S_{\text {osm(baseline) }}\right)^{K t}+S_{\text {osm(baseline) }} K=$ rate constant, tau $(\tau)=1 / K$, and $\tau_{1 / 2}=$ half-time $S_{\text {osm }}$ recovery. Data presented as mean \pm S.D 
Table 2 The return of $S_{\text {osm }}$ to baseline levels during rehydration

\begin{tabular}{|c|c|c|}
\hline & \multicolumn{2}{|c|}{ Recovery of Salivary Osmolality ( $\left.\mathrm{S}_{\mathrm{osm}}\right)$} \\
\hline & Females $(n=8)$ & Males $(n=9)$ \\
\hline \multicolumn{3}{|l|}{ Stimulated Sosm } \\
\hline \multicolumn{3}{|l|}{ Deep } \\
\hline $\mathrm{K}\left(\min ^{-1}\right)$ & $0.077 \pm 0.015$ & $0.123 \pm 0.077$ \\
\hline$\tau(\min )$ & $13.3 \pm 2.6$ & $10.5 \pm 4.3$ \\
\hline$\tau_{1 / 2}(\min )$ & $9.2 \pm 1.8$ & $7.2 \pm 3.0$ \\
\hline \multicolumn{3}{|l|}{ Sports } \\
\hline$K\left(\min ^{-1}\right)$ & $0.028 \pm 0.011$ & $0.035 \pm 0.021$ \\
\hline$\tau(\min )$ & $43.2 \pm 22.7$ & $38.5 \pm 23.8$ \\
\hline$\tau_{1 / 2}(\min )$ & $30.0 \pm 15.8$ & $26.6 \pm 16.5$ \\
\hline \multicolumn{3}{|l|}{ Spring } \\
\hline$K\left(\min ^{-1}\right)$ & $0.031 \pm 0.006$ & $0.032 \pm 0.023$ \\
\hline$\tau(\min )$ & $33.6 \pm 5.9$ & $43.9 \pm 19.6$ \\
\hline$\tau_{1 / 2}(\min )$ & $23.3 \pm 4.1$ & $30.4 \pm 13.6$ \\
\hline \multicolumn{3}{|c|}{ Unstimulated Sosm } \\
\hline \multicolumn{3}{|l|}{ Deep } \\
\hline $\mathrm{K}\left(\min ^{-1}\right)$ & $0.085 \pm 0.017$ & $0.106 \pm 0.0 .38$ \\
\hline$\tau(\min )$ & $12.3 \pm 2.7$ & $10.3 \pm 2.7$ \\
\hline$\tau_{1 / 2}(\min )$ & $8.5 \pm 1.8$ & $7.2 \pm 1.8$ \\
\hline \multicolumn{3}{|l|}{ Sports } \\
\hline$K\left(\min ^{-1}\right)$ & $0.028 \pm 0.013$ & $0.030 \pm 0.014$ \\
\hline$\tau(\min )$ & $42.0 \pm 16.6$ & $32.9 \pm 9.6$ \\
\hline$\tau_{1 / 2}(\min )$ & $29.1 \pm 11.5$ & $22.8 \pm 6.6$ \\
\hline \multicolumn{3}{|l|}{ Spring } \\
\hline$K\left(\min ^{-1}\right)$ & $0.028 \pm 0.011$ & $0.032 \pm 0.023$ \\
\hline$\tau(\min )$ & $42.1 \pm 13.3$ & $40.0 \pm 16.1$ \\
\hline$\tau_{1 / 2}(\min )$ & $29.1 \pm 19.1$ & $27.7 \pm 11.2$ \\
\hline
\end{tabular}

The relationship between $\mathrm{S}_{\mathrm{osm}}$ and time during the rehydration phase was best fit by a mono-exponential (one-phase decay) model. One-phase decay equation: Sosm $=($ Sosm(peak)- Sosm(baseline) $) \mathrm{Kt}+$ Sosm(baseline); $\mathrm{K}=$ rate constant, tau $(\tau)=1 / K$, and $\tau_{1 / 2}=$ half-time $S_{\text {osm }}$ recovery. Data presented as mean \pm S.D

\section{Dehydration, rehydration, and exercise performance}

Each participant (female and male) achieved a similar peak torque extension at baseline and immediately following $3 \%$ body mass loss across each study arm and is summarized in Fig. 5a. The dehydrating exercise protocol significantly impacted peak torque $(p=0.0048)$, which was dependent on sex $(p=0.0005)$. Overall, males generated greater peak torque extension at baseline when compared to females $(308.3 \pm 56.7 \mathrm{Nm}$ vs $172.8 \pm$ $40.8 \mathrm{Nm}$, respectively) and immediately following loss of $3 \%$ body mass $(276.3 \pm 39.5 \mathrm{Nm}$ vs $153.5 \pm 35.9 \mathrm{Nm}$, respectively). When comparing baseline and post-exercise peak torque, there was significant main effect of the exercise protocol on peak torque $(p=0.0002)$. However, the loss of peak torque reached significance only in males $(9.6+/-7.4 \% ; p=0.0117)$ and not in females $(10.6$
$+/-8.1 \% ; p=0.1998)$. Although participants in each study group failed to fully recover peak torque extension following rehydration, there was a significant effect of the rehydrating fluid ( $p=0.0157)$ on the percent peak torque recovery (Fig. 5b).

\section{Discussion}

The goal of the study was to evaluate parameters of dehydration and associated performance deficits due to dehydrating exercise, and then to determine if hydration and muscle performance recovery was dependent on fluid type. Secondarily, we observed potential sex differences in these parameters, although the study was not explicitly powered for such comparisons.

\section{Heart rate and body temperature}

Our observations on increases in heart rate are consistent with most [21-24], but not all [25] studies in the literature reporting statistically similar increases in heart rate for females and males during strenuous exercise. It has been suggested that males and females may differ in heart rate response to exercise, due in part to differences in exercise capacity, with men being able to reach higher exercise intensities, and therefore generate larger changes in heart rate during exercise [25]. It has also been suggested that a bias may exist in research personnel against pushing females as hard as males during exercise [25], and that males may put in a higher degree of effort during exercise than females [22], both of which could show confounded sex differences in peak heart rate. Indeed, we informally observed that males tended to exercise at a higher workload than females during our exercise study. However, our study, as well as another [22] showed similar max heart rates in females as males, despite the appearance of a difference in effort, indicating that males and females demonstrated similar exertion.

Sex differences may exist in thermoregulatory factors following heat stress and exercise [26], and we kept track of participants' tympanic temperature, which is representative of body temperature [27], throughout the exercise protocol. We observed a slight but statistically insignificant increase in tympanic temperature throughout the duration of the exercise protocol in men and women, with no differences between the sexes. This lack of difference between sexes was not surprising, because although males and females differ in some specific aspects of thermoregulation (sweat rate and evaporative cooling efficiency) during exercise in the heat, it is thought that females and males are able to maintain body temperature with similar efficiency [26]. However, we did not expect to see an overall lack of significant increase in body temperature after exercise, since much of the literature supports the idea that exercise, heat, and 

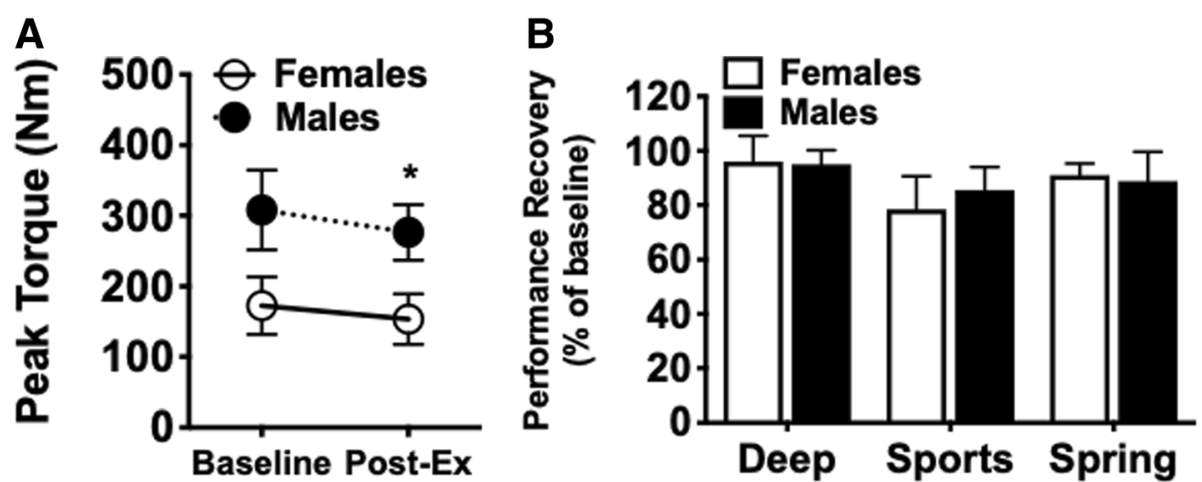

Fig. 5 Impact of dehydration and hydration on lower body muscle performance. Peak torque extension using Biodex ${ }^{\mathrm{TM}}$ was determined prior (Baseline) to and immediately following exercise (Post-Ex) and rehydrating protocol. a Averaged values across experimental groups for peak torque extension (Nm) at Baseline and Post-Ex in Females and Males. The dehydrating exercise protocol significantly impacted peak torque ( $p=0.0048)$, which was dependent on sex $(p=0.0005)$ and the exercise protocol ( $p=0.0002)$ by two-way ANOVA. Post-hoc Bonferroni analysis indicated a significant difference in Baseline and Post-Ex for Males only $(p=0.0117)$. the Deep group compared to both Sports and Spring groups $\left({ }^{*} p=0.01\right.$; Females vs. Males). $\mathbf{b}$ Bar graph representation of the recovery in peak torque extension at the completion of the hydration protocol expressed as percentage of baseline peak torque (Peak Torque end $_{\text {Peak Torque }}$ base $\left.X 100\right)$. There is a main effect of rehydrating fluid $(p=0.0157)$ by two-way ANOVA. Data presented as mean \pm S.D

dehydration impair thermoregulation [3, 11, 26]. One potential explanation for our data may be variation in the duration to achieve $3 \%$ body mass loss [3] or the fitness level of the study participants [3, 28]. More likely, acclimation to exercising in hot conditions may be the reason for this observation. Heat acclimation may provide the athlete with the benefit of expanded erythrocyte volume, and plasma volume, both of which have the potential to improve thermoregulatory ability in athletes [29]. We did not account for heat acclimation in this study, but it is reasonable to infer that some or all of the study participants had some level of heat acclimation living in Arizona, a region with a hot, dry climate throughout most of the year.

\section{Salivary osmolality and time to body mass loss}

Average baseline $\mathrm{S}_{\text {osm }}$ was not different between males and females. As expected, the total time to achieve a $3 \%$ reduction in body mass was similar among the three trials completed by each participant, regardless of sex, indicating consistency in exercise performance. Furthermore, we confirmed a significant positive correlation be tween percent body mass loss through sweat (dehydration) and $\mathrm{S}_{\text {osm }}$ for both males and females, as expected during intense exercise in the heat. These were important observations, because they indicate that participants started at the same hydration level and executed a similar amount of exercise during each trial. Although power output was not measured, we observed that men may have had higher average power output and tended to use greater resistance throughout the workout, consistent with findings showing higher aerobic workload capacity in men compared to women [30]. A higher power output in males could be one reason for the observed shorter time-to-dehydration than females. This difference in time-to-dehydration could also be attributed to a faster general sweat rate in males than in females, mainly due to greater body surface area and lower surface area-to-mass ratio, and greater metabolic heat production in males than in females [30, 31]. Although females generally have a greater number and density of eccrine sweat glands than men [30], the per-gland sweat secretion rate is a larger contributing factor to overall sweat rate than the number or density of sweat glands [31]. Sweat secretion rate per gland varies inter- and intra-individually, but it is possible that this factor may be partially responsible for this observed sweat rate difference.

\section{Dehydration, rehydration, and exercise performance}

Baseline and post-exercise values indicated that males generated greater peak torque than females, as expected, based on a higher average muscle mass in males than in females. In our study, fluid loss due to exercise resulted in a significant muscle performance deficit that was not impacted by sex. Although current literature is fairly inconclusive, results from many studies do suggest that dehydration negatively impacts muscular strength, power, and endurance [32]. However, there is relatively little research comparing potential dehydration-induced decline in muscle strength between men and women, and results of such studies vary. [32, 33]. The results of the current study do not necessarily support the notion that dehydration negatively impacts muscle strength, as the effects of dehydration were not isolated from the effects of exercise and muscle fatigue in this study. 
Interesting findings from previous work suggest that consumption of deep-ocean mineral water following a dehydrating exercise protocol improves aerobic performance and muscle strength $[13,14]$. Although these studies did not measure "hydration status", our initial study demonstrated a significantly more rapid return to pre-exercise (baseline) hydration levels in addition to improved recovery of lower body muscle performance following rehydration (post-hydration) with deep-ocean mineral water [15]. In this more comprehensive study, we found that male and female participants demonstrated elevated rates of hydration recovery, and that peak torque of a leg extension may also be improved when fluid was replenished with deep-ocean mineral water compared to other fluids. Therefore, improved acute hydration may be one factor by which deep-ocean mineral water improves exercise performance, as has been shown.

Although we did not study the precise mechanism underlying enhanced fluid recovery with deep-ocean mineral water, it is likely that the unique mineral composition of deep-ocean mineral water contributes to this characteristic (See Table 1 for a nutrient comparison of fluids). A 2013 study by Hou et al. [13] speculates that elevated levels of magnesium $(\mathrm{Mg})$ in deep-ocean mineral water may be a major contributor to its performance-enhancing effects, citing evidence of a correlation between $\mathrm{Mg}$ status and muscle strength. However, Kona Deep ${ }^{\circ}$ contains far less $\mathrm{Mg}$ than the deep-ocean mineral water used in the Hou study, and therefore, we cannot necessarily predict that the modest difference in $\mathrm{Mg}$ between the three fluids in our study was a major contributor to the observed effects on muscle performance. Additionally, we have no evidence to support a connection between $\mathrm{Mg}$ and hydration recovery.

Another possible mineral contributor is boron. Both Kona Deep and the water used in the Hou study contain significant amounts of this trace mineral. Hou reports that boron attenuates the rise in plasma lactate, potentially delaying fatigue, and prevents $\mathrm{Mg}$ loss. As with $\mathrm{Mg}$, however, we have no evidence to support a connection between boron and hydration recovery. Interestingly, composition of the intake fluid impacts intestinal water flux more so than osmolality [34]. Carbohydrate-electrolyte sports drinks, such as Gatorade $^{\circ}$, are proposed to increase intestinal water absorption due to the presence of glucose, which assists sodium transport into the intestinal cells via the sodium-glucose cotransporter, thereby influencing water flux by promoting an osmotic gradient $[35,36]$. However, we observed no greater acute hydration rate with Gatorade $^{\circ}$ compared to the other fluids. This may be due to the influence of gastric emptying rates, as fluids containing carbohydrates may decrease gastric emptying rate compared to non-carbohydrate-containing fluids [36, 37]. Notably, slower gastric emptying rates may also decrease intestinal absorption rates [35], thereby slowing overall fluid uptake and assimilation into the body fluid compartments.

\section{Limitations}

Several limitations of the study have been mentioned throughout the paper. We relied on the use of salivary osmolality as the sole marker of hydration throughout the study. Previous work shows that salivary osmolality is highly valuable for serial measures of hydration during intense physical activity in the heat [18]. More importantly, we needed multiple data points to best model instantaneous changes in osmolality throughout the dehydration and rehydration periods. Due to the continuous nature of the exercise protocol, serial urine collections were not practical for this study. Some limitations do exist for the use of $\mathrm{S}_{\text {osm }}$ as a marker of hydration, including an initial sharp drop in osmolality caused by oral rinse and variability between participants [3, 15, 17-19]. To account for these limitations, saliva samples were collected starting at $10 \mathrm{~min}$ after participants ingested the rehydrating fluid, which allowed $\mathrm{S}_{\text {osm }}$ to return to pre-rinse levels. Furthermore, baseline, peak or the rate of increase in $S_{\text {osm }}$ across the 3 trials was similar for each participant, indicating $\mathrm{S}_{\text {osm }}$ was an appropriate method for comparing rehydration fluids within each participant.

Participants were separated by sex based on secondary analysis of study parameters. Because the study was not powered for sex differences, analysis of peak torque would require further studies specifically powered for sex as a primary outcome. Similarly, dietary restrictions were suggested and not strictly enforced and cannot be ruled out as a potential contributor to any sex differences.

Finally, the American College of Sports Medicine (ACSM) recommends $1.25-1.5 \mathrm{~L}$ of replacement fluid for every $1 \mathrm{~kg}$ lost during exercise, as fluid loss continues post-exercise as a result of continued sweating and urinary losses [12]. In our study, participants replaced fluid lost in a 1:1 ratio. During development of the protocol in pilot studies, participants were not able to ingest fluid amounts suggested by the ACSM recommendations. In addition, participants did not urinate during rehydration, and all subjects completed the final saliva collection and muscle strength measurement at their full baseline body mass. Future studies will be designed to address these limitations as well as the underlying mechanisms by which deep-ocean mineral water elicited enhanced hydration effects, including the contribution of specific nutrients specific to deep-ocean mineral water. 


\section{Conclusions}

Kona Deep ${ }^{\circledR}$ deep-ocean mineral water improved acute rehydration rate after a dehydrating exercise in both males and females, compared to spring water and Gatorade ${ }^{\oplus}$ However, it remains unclear whether the hydration-enhancing effect of deep-ocean mineral water impacts performance recovery as demonstrated previously [13-15]. Future studies will be targeted at uncovering the mechanisms behind the hydration-enhancing properties of deep-ocean mineral water, further characterizing sex differences in these relationships, and correlating additional measures of hydration, such as serum osmolality, with that of $\mathrm{S}_{\mathrm{osm}}$.

\begin{abstract}
Abbreviations
BMI: Body Mass Index; bpm: Beats Per Minute; BSA: Body Surface Area; BW: Body Weight; CFTR: Cystic Fibrosis Transmembrane Protein; Deep: Kona Deep ${ }^{\oplus}$ Deep-Ocean Mineral Water; ECF: Extracellular Fluid; K: Rate Constant; Sosm: Salivary Osmolality; Sports: Gatorade ${ }^{\circledR}$ Sports Drink; Spring: Arrowhead Mountain Spring Water; UAHHSQ: University of Arizona Health History Screening Questionnaire; $\tau$ : Time Constant; $\tau_{1 / 2}$ : Time Constant Half-Life
\end{abstract}

\section{Acknowledgements}

We would like to thank Kona Deep ${ }^{\oplus \mathrm{Tm}}$ for providing the deep-ocean mineral water.

\section{Funding}

This work was supported by an Independent Scientist Award (K02 HL 105799) from the NIH and a grant from the Sarver Heart Center awarded to J.P. Konhilas. Support was provided by a Short Term Institutional Training Grant (T35HL007479-35) to E. Hines.

\section{Availability of data and materials}

The datasets used and/or analyzed during the current study are available from the corresponding author on reasonable request.

\section{Authors' contributions}

PRH oversaw completion of the exercise protocols, collected and analyzed participant data and saliva samples, and was the major contributor in writing the manuscript. DAK co-designed the study, supervised experiments in the exercise lab, and was a contributor in writing and revising the manuscript. EC oversaw completion of the exercise protocols, collected, analyzed, and interpreted participant data and saliva samples. SNW assisted with the exercise experiments, collected and analyzed saliva samples, and contributed to the revision of the manuscript. EH significantly contributed to the interpretation of the data, and the drafting and revision of the manuscript. MPK significantly contributed to the interpretation of the data, and the drafting and revision of the manuscript. ZIK acted as the responsible physician for the study, and significantly contributed to the drafting and revision of the manuscript. JPK co-designed the study, and supervised all research and statistical analysis of the data. All authors read and approved the final manuscript.

\section{Ethics approval and consent to participate}

All participants provided consent under protocols adhering to guidelines approved by the Institutional Review Board at the University of Arizona and in accordance with the Declaration of Helsinki.

\section{Consent for publication}

Not applicable.

\section{Competing interests}

1. Kona Deep ${ }^{\oplus \mathrm{\top} M}$ provided the deep-ocean mineral water.

2. The results of the present study do not constitute endorsement by ACSM

3. The results of the study are presented clearly, honestly, and without fabrication, falsification, or inappropriate data manipulation.

\section{Publisher's Note}

Springer Nature remains neutral with regard to jurisdictional claims in published maps and institutional affiliations.

\section{Author details}

${ }^{1}$ Department of Nutritional Sciences, University of Arizona, Tucson, AZ 85721, USA. ${ }^{2}$ Department of Physiology, University of Arizona, Tucson, AZ 85721 USA. ${ }^{3}$ Sarver Molecular Cardiovascular Research Program, University of Arizona, Tucson, AZ 85724, USA. ${ }^{4}$ Department of Surgery, University of Arizona, Tucson, AZ 85721, USA.

Received: 5 September 2018 Accepted: 19 March 2019

Published online: 04 April 2019

\section{References}

1. Bhave G, Neilson EG. Body fluid dynamics: back to the future. J Am Soc Nephrol. 2011;22(12):2166-81.

2. Bourque $\mathrm{CW}$. Central mechanisms of osmosensation and systemic osmoregulation. Nat Rev Neurosci. 2008;9(7):519-31.

3. Cheuvront SN, Carter R 3rd, Sawka MN. Fluid balance and endurance exercise performance. Curr Sports Med Rep. 2003;2(4):202-8.

4. Shibasaki M, Wilson TE, Crandall CG. Neural control and mechanisms of eccrine sweating during heat stress and exercise. J Appl Physiol (1985). 2006:100(5):1692-701.

5. Lara B, Salinero JJ, Areces F, Ruiz-Vicente D, Gallo-Salazar C, Abian-Vicen J, Del Coso J. Sweat sodium loss influences serum sodium concentration in a marathon. Scand J Med Sci Sports. 2017;27(2):152-60.

6. Sawka MNWCB, Pandolf KB. Thermoregulatory responses to acute exerciseheat stress and heat acclimatization. In: Fregly MJBCM, editor. Handbook of physiology. New York: Oxford University Press; 1996. p. 157-85.

7. Fisher SK, Heacock AM, Keep RF, Foster DJ. Receptor regulation of osmolyte homeostasis in neural cells. J Physiol. 2010;588(Pt 18:3355-64.

8. Sawka MN, Young AJ, Francesconi RP, Muza SR, Pandolf KB. Thermoregulatory and blood responses during exercise at graded hypohydration levels. J Appl Physiol (1985). 1985;59(5):1394-401.

9. Armstrong $L E$, Costill $D L$, Fink WJ. Influence of diuretic-induced dehydration on competitive running performance. Med Sci Sports Exerc. 1985;17(4):456-61.

10. Maughan RJ, Shirreffs SM. Dehydration and rehydration in competitive sport. Scand J Med Sci Sports. 2010;20(Suppl 3):40-7.

11. Sawka MN, Cheuvront SN, Kenefick RW. Hypohydration and human performance: impact of environment and physiological mechanisms. Sports Med. 2015;45(Suppl 1):51-60.

12. Thomas DT, Erdman KA, Burke LM. American College of Sports Medicine joint position statement. Nutrition and athletic performance. Med Sci Sports Exerc. 2016;48(3):543-68.

13. Hou CW, Tsai YS, Jean WH, Chen CY, Ivy JL, Huang CY, Kuo CH. Deep ocean mineral water accelerates recovery from physical fatigue. J Int Soc Sports Nutr. 2013;10(1):7

14. Wang ML, Chen YJ, Cheng FC. Nigari (deep seawater concentrate) enhances the treadmill exercise performance of gerbils. Biol Sport. 2014;31(1):69-72.

15. Keen DA, Constantopoulos E, Konhilas JP. The impact of post-exercise hydration with deep-ocean mineral water on rehydration and exercise performance. J Int Soc Sports Nutr. 2016;13:17.

16. Galloway SD, Maughan RJ. Effects of ambient temperature on the capacity to perform prolonged cycle exercise in man. Med Sci Sports Exerc. 1997; 29(9):1240-9.

17. Ely BR, Cheuvront SN, Kenefick RW, Spitz MG, Heavens KR, Walsh NP, Sawka MN. Assessment of extracellular dehydration using saliva osmolality. Eur J Appl Physiol. 2014;114(1):85-92.

18. Munoz CX, McKenzie AL, Armstrong LE. Optimal hydration biomarkers: consideration of daily activities. Obes Facts. 2014;7(Suppl 2):13-8.

19. Cheuvront SN, Ely BR, Kenefick RW, Sawka MN. Biological variation and diagnostic accuracy of dehydration assessment markers. Am J Clin Nutr. 2010;92(3):565-73.

20. Du Bois D, Du Bois EF. A formula to estimate the approximate surface area if height and weight be known. 1916. Nutrition. 1989;5(5):303-11; discussion 312-303.

21. Cote AT, Phillips AA, Foulds HJ, Charlesworth SA, Bredin SS, Burr JF, Koehle MS, Warburton DE. Sex differences in cardiac function after prolonged strenuous exercise. Clin J Sport Med. 2015;25(3):276-83. 
22. Fomin A, Ahlstrand M, Schill HG, Lund LH, Stahlberg M, Manouras A, Gabrielsen A. Sex differences in response to maximal exercise stress test in trained adolescents. BMC Pediatr. 2012;12:127.

23. Itoh H, Ajisaka R, Koike A, Makita S, Omiya K, Kato Y, Adachi H, Nagayama M, Maeda T, Tajima A, et al. Heart rate and blood pressure response to ramp exercise and exercise capacity in relation to age, gender, and mode of exercise in a healthy population. J Cardiol. 2013;61(1):71-8.

24. Maruf FA, Ogochukwu UN, Dim PA, Alada AR. Absence of sex differences in systolic blood pressure and heart rate responses to exercise in healthy young adults. Niger J Physiol Sci. 2012;27(1):95-100.

25. Sydo N, Abdelmoneim SS, Mulvagh SL, Merkely B, Gulati M, Allison TG Relationship between exercise heart rate and age in men vs women. Mayo Clin Proc. 2014;89(12):1664-72.

26. Kaciuba-Uscilko H, Grucza R. Gender differences in thermoregulation. Curr Opin Clin Nutr Metab Care. 2001:4(6):533-6.

27. Fogt $\mathrm{DL}$, Henning AL, Venable AS, MCFarlin BK. Non-invasive measures of Core temperature versus ingestible thermistor during exercise in the heat. Int J Exerc Sci. 2017;10(2):225-33.

28. Judelson DA, Maresh CM, Farrell MJ, Yamamoto LM, Armstrong LE, Kraemer WJ, Volek JS, Spiering BA, Casa DJ, Anderson JM. Effect of hydration state on strength, power, and resistance exercise performance. Med Sci Sports Exerc. 2007;39(10):1817-24.

29. Sawka MN, Coyle EF. Influence of body water and blood volume on thermoregulation and exercise performance in the heat. Exerc Sport Sci Rev. 1999;27:167-218.

30. Kenney WL. A review of comparative responses of men and women to heat stress. Environ Res. 1985;37(1):1-11.

31. Baker LB. Sweating rate and sweat sodium concentration in athletes: a review of methodology and intra/Interindividual variability. Sports Med. 2017;47(Suppl 1):111-28.

32. Judelson DA, Maresh CM, Anderson JM, Armstrong LE, Casa DJ, Kraemer WJ, Volek JS. Hydration and muscular performance: does fluid balance affect strength, power and high-intensity endurance? Sports Med. 2007;37(10):907-21.

33. Gutierrez A, Mesa JL, Ruiz JR, Chirosa LJ, Castillo MJ. Sauna-induced rapid weight loss decreases explosive power in women but not in men. Int J Sports Med. 2003;24(7):518-22.

34. Gisolfi CV, Lambert GP, Summers RW. Intestinal fluid absorption during exercise: role of sport drink osmolality and [ $\mathrm{Na}+]$. Med Sci Sports Exerc. 2001;33(6):907-15.

35. Leiper JB. Fate of ingested fluids: factors affecting gastric emptying and intestinal absorption of beverages in humans. Nutr Rev. 2015;73(Suppl 2): $57-72$.

36. Murray R. The effects of consuming carbohydrate-electrolyte beverages on gastric emptying and fluid absorption during and following exercise. Sports Med. 1987:4(5):322-51.

37. Murray R, Bartoli W, Stofan J, Horn M, Eddy D. A comparison of the gastric emptying characteristics of selected sports drinks. Int J Sport Nutr. 1999;9(3): 263-74.

Ready to submit your research? Choose BMC and benefit from:

- fast, convenient online submission

- thorough peer review by experienced researchers in your field

- rapid publication on acceptance

- support for research data, including large and complex data types

- gold Open Access which fosters wider collaboration and increased citations

- maximum visibility for your research: over $100 \mathrm{M}$ website views per year

At $\mathrm{BMC}$, research is always in progress.

Learn more biomedcentral.com/submissions 